\section{Efficacy of incisional vs punch biopsy in the histological diagnosis of periocular skin tumours}

JC Rice' ${ }^{1}$, P Zaragoza' ${ }^{1}$, K Waheed' , J Schofield' and CA Jones
${ }^{1}$ Kent County Ophthalmic Hospital

Maidstone, Kent

Correspondence:

Dr JC Rice

Department of

Ophthalmology

Medical School

Observatory

7925 Cape Town

South Africa

Tel: + 2721406 6215/6

Fax: + 27214066218

E-mail: ricejs@

metroweb.co.za

Received: 19 March 2002 Accepted: 20 August 2002

\section{Abstract}

Aims The aim of this study was to compare the accuracy of incisional and punch biopsy techniques in obtaining correct histological diagnosis of periorbital eyelid tumours. The technique of punch biopsy is presented and described in detail.

Methods A retrospective analysis was made of 20 consecutive incisional biopsies and 20 consecutive punch biopsies. In each case, the histology obtained at biopsy was compared with that identified at the time of tumour excision.

Results A total of 40 consecutive biopsies on 38 patients were analysed. The first 20 were incisional; the second 20 were punch biopsies. Of the 20 incisional biopsy specimens, 19 were confirmed accurate at the time of excision of the lesion. Of the 20 punch biopsies, 17 were confirmed accurate at the time of excision. These correspond to accuracy rates of 95 and $85 \%$, respectively.

Conclusions Both incisional and punch biopsy techniques have relatively high accuracy rates and there is a high concordance between tissue diagnoses made by each of these techniques. Incisional techniques should preferably be performed on any atypical lesion. Punch biopsy is a quick and simple procedure. It is easy to perform in an outpatient environment and requires a minimum of surgical equipment and no specific surgical skills. If the site of biopsy is carefully chosen, this simple technique provides tissue specimens of adequate size and quality for accurate histology and is a most useful adjunct in the management of periocular tumours.

Eye (2003) 17, 478-481. doi:10.1038/

sj.eye. 6700383
Keywords: biopsy technique; histology; incisional biopsy; punch biopsy

Introduction

When planning tumour excision and reconstruction, it is essential to obtain histological diagnosis of the lesion. While excisional biopsy may be practical in some small lesions, an incisional biopsy is usually performed at the edge of a lesion prior to its excision. We report an alternative biopsy technique, the punch biopsy, and compare the accuracy of these two biopsy techniques in diagnosing periorbital eyelid tumours.

Methods

This study is a retrospective analysis of patients who have undergone initial biopsy and subsequent excision of lid tumours between 1994 and 2000. We analysed 20 consecutive incisional biopsies and 20 consecutive punch biopsies, and compared the histology obtained with that identified at the time of tumour excision.

\section{Technique of punch biopsy}

Punch biopsy is a simple, quick procedure that can easily be performed in the outpatient clinic and can readily be mastered by any member of the ophthalmic team. Warren et $a l^{1}$ describe the technique using a 3-4 mm punch. The biopsy site then required closure with one or two sutures. We suggest using a $2 \mathrm{~mm}$ punch which removes the need for sutures. 


\section{Typical biopsy pack (Figure 1)}

The Typical biopsy pack comprises the following:

(1) $2 \mathrm{~mm}$ disposable punch,

(2) fine-toothed forceps,

(3) spring scissors,

(4) disposable cautery.

\section{Biopsy site}

Many periocular lesions develop central areas of keratinisation. These areas are best avoided as the histology in this area is often inconclusive. The junction between the lesion and the surrounding skin should also be avoided as biopsy at this site disrupts the margin of the lesion, making the histological diagnosis of tumour clearance more difficult. The best site for biopsy is normally a more marginal region of the lesion, but not too close to its visible edge. A single tissue sample is usually adequate, but if uncertainty exists about the quality of one specimen, then further biopsies should be taken from other sites.

\section{Technique}

The patient is best positioned semirecumbent in a reclining chair. A small amount of local anaesthetic with adrenaline $1: 100000$ is injected beneath the lesion, being careful not to enter the lesion as this may disrupt its margin and make the histological diagnosis of clearance more difficult. The $2 \mathrm{~mm}$ punch is placed over a suitable area of the lesion and twisted in a circular manner with

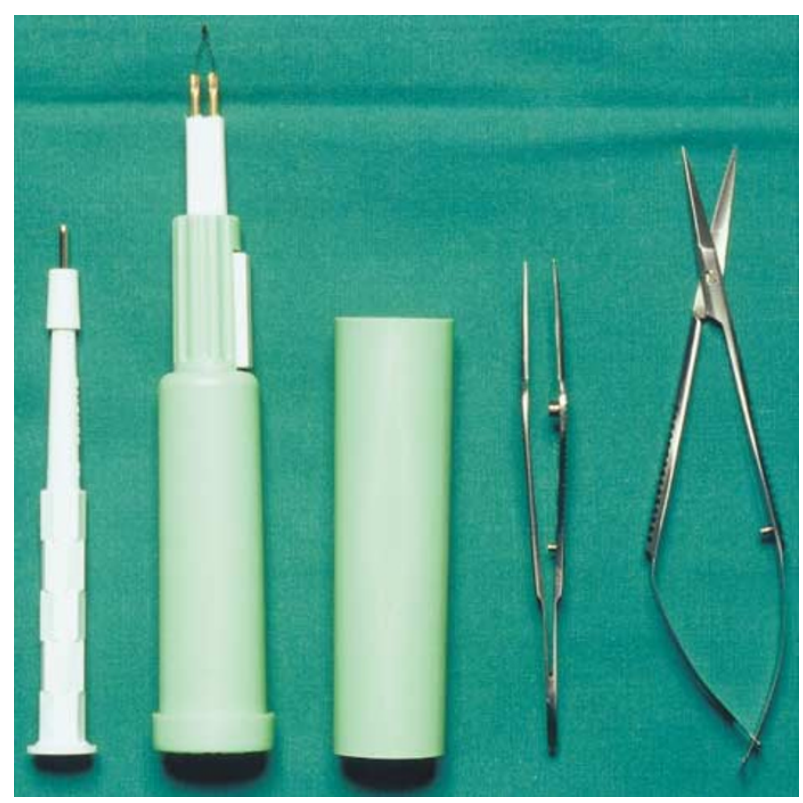

Figure 1 Biopsy pack. gentle downward pressure so that the tip enters the substance of the lesion. Care needs to be taken not to push the punch too far into the lesion as full-thickness lid penetration can occur putting the globe at risk. The punch is carefully removed perpendicular to the skin so as not to tear off the biopsy specimen which remains in situ.

The edge of the specimen is then grasped with the forceps, being careful not to cause significant crush artefact. Sassani ${ }^{2}$ describes the use of a traction suture passed through the base of the specimen at this stage. This provides traction and eliminates the need to grasp the lesion with forceps. In our series, crush artefact was not a significant complication. We suggest the careful use of forceps, which simplifies the procedure. The specimen is gently lifted out of the surrounding tissue and the base is cut with spring scissors.

Haemostasis is achieved with gentle cautery to the base of the wound. No suturing is necessary if a $2 \mathrm{~mm}$ punch is used. A small dry dressing may be applied.

\section{Results}

In all, 40 consecutive biopsies on 38 patients were analysed. The first 20 of these were incisional; and the second 20 punch biopsies.

\section{Incisional biopsies}

Of the 20 specimens, 16 were diagnosed as basal cell carcinomas (BCCs), two squamous cell carcinomas (SCCs), one intradermal naevus, and one chronic inflammation. Of these, 19 were confirmed accurate at the time of excision of the lesion. The biopsy that revealed chronic inflammation was found to be a BCC when excised. The results are summarised in Table 1.

\section{Punch biopsies}

In total, 20 punch biopsies were undertaken. Of them, 16 were diagnosed as BCCs, one sebaceous gland carcinoma, one hyperkeratosis, and two actinic keratosis. In three cases, the excision histology was different from that obtained at punch biopsy. The patient with hyperkeratosis was found to have a BCC. Both patients with actinic keratosis at the time of punch biopsy were also inaccurate. One proved to be an SCC and the other a BCC. The results are summarised in Table 2.

In our findings, the accuracy rates of incisional and punch biopsies were 95 and 85\%, respectively.

\section{Discussion}

Numerous benign and malignant tumours occur on the eyelids. A large study of 892 lid lesions examined over a 38-year period between 1932 and 1969 found malignant 
tumours in $24 \%$ of cases. ${ }^{3}$ BCC is by far the most common malignancy of the eyelids and canthi constituting $80-95 \%$ of all malignancies in this region. ${ }^{4,5}$ SCC is the second most common periorbital malignancy (9.2\%). Less common are sebaceous gland carcinoma (1-6.4\%), malignant melanoma, eccrine and apocrine sweat gland tumours, Merkel cell tumours, malignant lymphomas, and metastasis from other sites.

The accurate diagnosis and correct management of these tumours requires histological diagnosis, which is usually obtained by biopsy. Incisional biopsy, punch biopsy, and cytological examination of scrapings from the lesion are all well-described techniques. Of these, incisional biopsy is consistently recognised as the gold standard. While it is relatively easy to perform, a certain degree of surgical skill is necessary and the procedure is time consuming.

Our small series demonstrated that incisional biopsy produces a more accurate assessment of the tumour than punch biopsy. We did, however, find one out of 20 incisional biopsies to be inaccurate. This represents a failure rate of $5 \%$. Of the punch biopsy group, three out of 20 were misdiagnosed at the time of biopsy, hence a higher failure rate of $15 \%$.

Punch biopsy has the advantage of being a quick technique requiring a minimum of equipment. In addition, the operator requires no specific surgical skills. The biopsy specimen can easily be taken at the patient's initial clinic visit allowing a more rapid diagnosis and facilitating more efficient tumour management and fewer visits to hospital. Previous description ${ }^{1}$ of the technique has highlighted the excellent specimens obtained by this method, which is widely used in dermatological practice.

Specimens obtained by punch biopsy compare very favourably with those obtained by incisional biopsy. Todd $e t a l^{6}$ reported a $94 \%$ concordance rate between

Table 1 Results of incisional biopsy vs final histology

\begin{tabular}{ll}
\hline Incisional biopsy & Final histology \\
\hline 16 BCC & 16 BCC \\
2 SCC & 2 SCC \\
1 intradermal naevus & Intradermal naevus \\
1 chronic inflammation & BCC \\
\hline
\end{tabular}

Table 2 Results of punch biopsy vs final histology

\begin{tabular}{ll}
\hline Punch biopsy & Final histology \\
\hline 16 BCC & 16 BCC \\
1 sebaceous gland carcinoma & 1 sebaceous gland carcinoma \\
1 hyperkeratosis & BCC \\
2 actinic keratosis & $1 \mathrm{BCC}, 1$ SCC \\
\hline
\end{tabular}

$2 \mathrm{~mm}$ punch biopsies and ellipses, where the punch was taken from the centre of the ellipse and analysed first. They suggest that concordance is even higher (96\%) if haemangiomas and other vascular lesions are electively not punch biopsied, as these specimens are too small to contain enough supportive tissue for the ectatic blood vessels.

Russell $\mathrm{et} \mathrm{al}^{7}$ demonstrated an $80.7 \%$ (46 out of 57 specimens) accuracy rate in diagnosing histological subtypes of BCC from punch biopsy specimens. They did not record the size of the punch biopsies used in their study, but their results again support this technique of tumour biopsy as a reliable method of making an accurate tissue diagnosis. Our study suggests that the $2 \mathrm{~mm}$ punch biopsy technique yields specimens of adequate size and quality to allow an accurate histological diagnosis to be made, although in our series the procedure was only $85 \%$ accurate. We believe that this accuracy rate can be improved if the site of biopsy is carefully chosen and areas of hyperkeratosis are avoided.

Barton $e t l^{8}$ suggest that cytology may be sufficiently accurate to plan excision and reconstructive surgery, particularly for BCCs. This technique shares the advantages of the punch biopsy in that it is quick and easy to perform. One of its main limitations is that tissue architecture is not reliably preserved, making it less suitable for diagnosing lesions in which accurate assessment of morphology is important. In this setting, incisional and punch biopsies are more reliable, particularly if a lesion other than a BCC is suspected.

\section{Conclusion}

In conclusion, both incisional and punch biopsy techniques have relatively high accuracy rates and there is a high concordance between tissue diagnoses made by each of these techniques. Incisional techniques have, however, proved more accurate in identifying the true nature of a tumour. We would encourage incisional biopsies to be performed on any atypical lesion, but the technique of punch biopsy should not be ignored. If the site of biopsy is carefully chosen, this simple technique is a most useful adjunct in the management of periocular tumours.

\section{Acknowledgements}

The suppliers of the biopsy punch are Stiefel Laboratories Ltd (Holtspur Lane, Wooburn Green, Bucks HP10 0AU, UK). The authors have no proprietary interest in this product. 


\section{References}

1 Warren RC, Nerad JA, Carter KD. Punch biopsy technique for the ophthalmologist (letter). Arch Ophthalmology 1990; 108(6): 778-779.

2 Sassani JW. Punch biopsy technique for the ophthalmologist (letter; comment). Arch Ophthalmol 1991; 109(4): 464-465.

3 Aurora AL, Blodi FC. Lesions of the eyelids: a clinicopathological study. Surv Ophthalmol 1970; 15: 94.

4 Mamalis N, White Jr GL, Pedersen DM, Holds J, Anderson RL. Malignant lesions of the eyelids. Am Fam Physician 1989; 39: 95-102.
5 Francis IC, Benecke PS, Kappagoda MB. A ten-year hospital survey of eyelid cancer. Aust J Ophthalmol 1984; 12(2): 121-127.

6 Todd P, Garioch JJ, Humphreys S, Seywright M, Thomson J, $\mathrm{du}$ Vivier AW. Evaluation of the 2-mm punch biopsy in dermatological diagnosis. Clin Exp Dermatol 1996; 21(1): 11-13.

7 Russell EB, Carrington PR, Smoller BR. Basal cell carcinoma: a comparison of shave biopsy versus punch biopsy techniques in subtype diagnosis. J Am Acad Dermatol 1999; 41(1): 69-71.

8 Barton K, Curling OM, Paridaens AD, Hungerford JL. The role of cytology in the diagnosis of periocular basal cell carcinomas. Ophthalmic Plast Reconstr Surg 1996; 12(3): 190-194, discussion 195. 